\title{
Research progress of restraining campus bullying by martial arts training
}

\author{
Ting Xu \& Guodong Zhang* \\ College of Physical Education, Southwest University, Chongqing (China)
}

8th IMACSSS International Conference Abstracts, Viseu (Portugal), October 10-12, 2019

Type: Poster presentation

\begin{abstract}
This narrative review analyzed the existing research related to the practice of martial arts and campus bullying. This literature suggests that martial arts training can reduce bullying behaviors by improving individual self-control and self-esteem, reducing hostility and strengthening interpersonal communicative ability. Current research also emphasizes the efficacy of those martial arts activities that pay attention to participants' moral education or norms of proper social behavior in order to improve physical cognition and lead to restraining individual bullying behavior. Future research should focus on cross-cultural factors and local situations, combine quantitative and qualitative research methods, and explore the internal mechanism of martial arts training to curb campus bullying, so as to explore new ways in which martial activity can promote individual psychological adjustment and to positively address problem behavior.
\end{abstract}

Keywords: Martial arts; combat sports; martial arts training; bullying; violence prevention.

\section{Introduction}

In recent years, the phenomenon of bullying has been documented around the world (Qiao, Xing, Ji, \& Zhang, 2009; Masilo, 2018). Campus bullying takes a great toll on individuals' physical and mental health, and it is not uncommon for teenagers to commit suicide as a result of being bullied. Therefore, campus bullying has attracted wide attention across all sectors of society, with most academic studies focusing on psychology, education or sports. Studies have found that physical exercise plays a significant role in developing individual self-control and behavioral restraint. Physical exercise can reduce individuals' negative emotions and make them more inclined to adopt positive coping methods when facing problems, thus reducing the generation of negative emotions and behaviors. Martial arts, including both sparring and the practice of prearranged actions (e.g., kata, sets) are an international phenomenon existing in culture specific forms (Liu, 2007). Campus bullying, like martial arts, knows no cultural boundaries and is a behavioral pattern influenced by individual cognition. Both are physical activities generated by individual ideology. Most people practice martial arts to strengthen their bodies and build their characters, but the essence of martial arts is attack and defence, and the practice of offensive and defensive techniques affects individual behaviors. Bandura's social learning theory argues that individual behaviors are generated in the process of social learning (Bandura, 1973). Practicing attack and defence can help individuals have a deeper understanding of martial arts, but moral constraints are required to channel individual behaviors into positive rather than negative results. Therefore, in the campus environment, does martial arts practice inhibit or breed bullying behavior? The purpose of this study is to review the existing mechanisms of martial arts practice to curb campus bullying.

\section{Methodology}

This narrative review searched for key terms related to the martial arts and bullying, such as "martial arts", "combat sport", "bullying", and "violence", in CNKI (China National Knowledge Infrastructure), Google and Web of Science databases. Resources for the past 20 years on these 
topics were reviewed from the psychological perspective in order to understand the inner link between bullying and martial arts practice. The literature content analysis method was followed for extracting and syntetizing data from the selected references.

\section{Results}

Most bullies manifest poor self-restraint. Research establishes that taking part in martial arts training improves general self-control, and martial arts practice can also curb aggressive behavior. Therefore, as regards to controlling campus bullying, martial arts intervention can improve individual self-restraint, establish socially correct values and morality, and substantially reduce individual violence and anti-social behavior (White \& Turner, 2014).

As an active way of life, martial arts practice can not only strengthen the body, but also improve self-concept and self-esteem. Campus bullies attempt to increase self-confidence by dominating peers through aggressive behavior. Individuals involved in campus bullying typically demonstrate low self-confidence, low self-esteem and, in the case of victims, are easily intimidated, and bullied by others in social interactions. Through martial arts practice, individuals can enhance self-confidence and self-esteem, thus gaining more respect in daily life (Fabio \& Towey, 2018).

During social interactions, bullies commonly use negative coping styles to solve problems when dealing with stressful events because they cannot control their anger and similar negative emotions, thus intensifying the occurrence of campus bullying. Martial arts practice can direct individuals from negative behavior by increasing their positive emotions and mindsets (Fung \& Lee, 2018). In addition, the hypothesis of social interaction suggests that physical exercise can enhance social interaction with classmates, friends, and improve mental health (North, McCullagh, \&Tran, 1990). Martial arts practice can lead to the acquisition of a circle of martial friends, and provide opportunities for positive interactions through communication and exchange with others sharing the same interests.

\section{Discussion and Conclusion}

Studies found that campus bullying is often an externalized problem behavior reflecting the condition of poor individual self-control and impaired psychological adjustment functions, seriously hindering the student's healthy physical and mental development. Combined theoretical analysis and experimental research found a lack of direct intervention and related research on martial arts training to curb campus bullying. Most research emphasizes employing martial arts training to regulate individual behavior and, thus, indirectly curb campus bullying. Martial arts exist within specific cultural contexts, each with their own ideologies and worldviews. Therefore, it remains for further studies to determine whether there are cross-cultural differences in the influences of martial arts practice on behaviors and whether these influences on martial arts training on individual behaviors differ. Future research should take into account the influence of such localized characteristics on behavior to further explore the internal mechanism of martial arts training to curb campus bullying from multiple perspectives.

\section{References}

Bandura, A. (1973). Aggression: A social learning analysis. American Journal of Sociology, 26(5), 1101-1109. doi: $10.2307 / 1227918$

Fung, A. L. C., Lee, T. K. H. (2018). Effectiveness of chinese martial arts and philosophy to reduce reactive and proactive aggression in schoolchildren. Journal of Developmental \& Behavioral Pediatrics, 39(5), 404-414. doi: 10.1097/DBP.0000000000000565

Fabio, R. A., Towey, G. E. (2018). Cognitive and personality factors in the regular practice of martial arts. The Journal of sports medicine and physical fitness, 58(6), 933-943. doi: 10.23736/S0022-4707.17.07245-0

Liu, C. P. (2007). The argumentum of the development of wushu in home and abroad. Journal of Jilin Institute of Physical Education, 23(6), 161-162.

Masilo, D. T. (2018). Social work intervention to address the phenomenon of bullying amongst learners in the school setting: A literature review. South African Journal of Education, 38(1), 1-9. doi: 10.15700/saje.v38ns1a1594 
North, T. C., McCullagh, P., \&Tran Z. V. (1990). Effect of exercise on depression. Exercise \& Sport Sciences Reviews, 18(1), 379-416. doi: 10.1249/00003677-199001000-00016

Qiao, Y. J., Xing, Y., Ji, C. Y., \& Zhang, L. (2009). The prevalence of bullying behaviors among urban middle school students in 18 provinces, china. Chinese Journal of Epidemiology, 30(5), 444447. doi: $10.3760 /$ cma.j.jissn.0254-6450.2009.05.007

White, B. A., Turner, K. A. (2014). Anger rumination and effortful control: mediation effects on reactive but not proactive aggression. Personality and Individual Differences, 56,186-189. doi: $10.1016 /$ j.paid.2013.08.012 